\title{
Effect of Adrenaline and Guanethidine in reducing intraocular pressure in rabbits' eyes
}

\author{
D. E. P. JONES*, D. A. NORTON, JUDITH HARVEY, AND D. J. G. DAVIES \\ From the School of Pharmacy and Pharmacology, University of Bath, and *Royal United Hospitals, Bath
}

Adrenaline (Epinephrine) and Guanethidine (Ismelin), apart from supplementing the effect of Pilocarpine, are used in the treatment of open-angle glaucoma both alone and in combination, one with the other. The mechanisms of their individual and combined activity in reducing intraocular pressure (IOP), however, are still not fully understood.

Langham, Kitazawa, and Hart (197I) have pointed out that in animal eyes the presence has been established of adrenergic mechanisms that are capable of both increasing and decreasing blood flow to the microcirculatory system linking the ciliary processes through which aqueous humour is formed, and to the intrascleral and episcleral systems through which both the aqueous humour and intraocular blood are drained. In man evidence for a similar dual mechanism is less convincing. Adrenaline (Epinephrine), a catecholamine having both alpha- and beta- adrenergic agonistic properties, decreases intraocular pressure when applied topically in low concentrations without an increase in outflow facility and without pupil dilatation, but at higher concentrations it increases outflow facility and mydriasis takes place. Noradrenaline (Norepinephrine), the alpha-adrenergic agonist, has also been reported by Duke-Elder (1962) to decrease intraocular pressure but to be less effective than Adrenaline.

Guanethidine on the other hand is a post-ganglionic adrenergic neurone-blocking drug, its antihypertensive effect being due to the impairment of release of Noradrenaline from adrenergic nerve junctions with vascular smooth muscles, the reduction obtained in the intraocular pressure being related to decreased production of the aqueous humour, in addition to its miotic effect improving the facility of aqueous outflow. However, in addition to its blocking effect on sympathetic adrenergic activity, it has been postulated that Guanethidine sensitizes receptor cells in the ciliary body and ciliary processes to catecholamines generally and that application of Guanethidine together with Adrenaline will be more

Address for reprints: D. J. G. Davies, School of Pharmacy and Pharmacology, University of Bath, Bath effective in reducing intraocular pressure than would either drug alone. This has been indicated by the work of Roth (1973), Turner (1968), and Paterson and Paterson (1972) on unpublished results of Crombie.

Guanethidine and Adrenaline are marketed as individual solutions and are generally used in this way. In order to prevent the washing away of the active drug a time lapse is often advocated between instillation of the two formulations. One of us (DEPJ), using an interval of $30 \mathrm{~min}$, has shown over a 2-year period that IOP in glaucomatous patients can be adequately controlled by this dosage regimen. If the postulated Guanethidine sensitization is rapidly achieved then it would be advantageous to patients if both drugs were to be combined in one formulation and so instilled at the same time.

Rabbits demonstrate a measurable decrease in IOP as a result of instillation into the eye of Adrenaline or Guanethidine. Rabbits were therefore used in this study which was aimed at elucidating the relationship between effectiveness and dosage protocol of Guanethidine and Adrenaline.

\section{Material and methods}

Three different eye drop formulations were used.

(I) Adrenaline hydrogen tartrate 0.5 per cent $\mathrm{w} / \mathrm{v}$ in Kalthoff's borate-phosphate buffer at $\mathrm{pH} 7 \cdot 4$ containing 0.01 per cent $\mathrm{w} / \mathrm{v}$ benzalkonium chloride and $\mathrm{o} \cdot \mathrm{I}$ per cent $\mathrm{w} / \mathrm{v}$ sodium metabisulphite.

(2) Guanethidine sulphate 2.5 per cent $w / v$ in the same buffer at $\mathrm{pH} 7 \cdot 4$ containing $0 \cdot 0$ or per cent $\mathrm{w} / \mathrm{v}$ benzalkonium chloride and $o \cdot 1$ per cent $w / v$ sodium metabisulphite.

(3) Adrenaline hydrogen tartrate 0.5 per cent $w / v$ and Guanethidine sulphate 2.5 per cent $w / v$ in the same buffer at 7.4 containing 0.01 per cent $w / v$ benzalkonium chloride and $o \cdot I$ per cent $w / v$ sodium metabisulphite.

All solutions were prepared with freshly boiled and cooled distilled water. They were sterilized by filtration through a $0.22 \mu$ membrane filter.

The experimental animals were six male New Zealand white rabbits aged between 4 and 8 months. They were conditioned to having eye drops instilled into their eyes 
and having their IOP measured daily for one month before the experiment began.

Intraocular pressure measurements were obtained with a Perkins hand-held applanation tonometer using benoxinate as a local anaesthetic before the measurements were taken. The rabbits were placed in a holding box whilst IOP measurements were made.

For the assessment of activity of the different dosage protocols on each day the rabbits were divided into three groups of two. Both members of each group were given one drop of the test formulation/s in one eye and a drop of sterile saline in the other. IOP measurements were then performed on both eyes at regular intervals until the IOP in the test eye had returned to the same value as the control eye. On each occasion the same groupings were used twice, on the first day the left eye being used as the test and on the second day the right eye. The rabbits were allowed 3 clear days between each experiment so that in each week three dosage protocols were tested, each on two rabbits. The normal crossover procedure was applied so that in the second and third week the rabkits were subjected to the two other dosage protocols. Thus by the end of 3 weeks all six rabbits had received drugs in each eye according to three different dosage protocols. This scheme is outlined in Table I.

Table I Experimental design for testing three different dosage protocols

\begin{tabular}{|c|c|c|c|c|}
\hline \multirow[b]{2}{*}{ Week } & \multirow[b]{2}{*}{ Day } & \multicolumn{3}{|c|}{ Dosage Protocol } \\
\hline & & Group I & Group 2 & Group 3 \\
\hline 1 & $\begin{array}{l}1 \\
2\end{array}$ & $\begin{array}{l}\mathrm{Ar} \\
\mathrm{Al}\end{array}$ & $\begin{array}{l}\mathrm{Br} \\
\mathrm{Bl}\end{array}$ & $\begin{array}{l}\mathrm{Cr} \\
\mathrm{Cl}\end{array}$ \\
\hline 2 & $\begin{array}{l}1 \\
2\end{array}$ & $\begin{array}{l}\mathrm{Cr} \\
\mathrm{Cl}\end{array}$ & $\begin{array}{l}\mathrm{Ar} \\
\mathrm{Al}\end{array}$ & $\begin{array}{l}\mathrm{Br} \\
\mathrm{Bl}\end{array}$ \\
\hline 3 & $\begin{array}{l}1 \\
2\end{array}$ & $\begin{array}{l}\mathrm{Br} \\
\mathrm{Bl}\end{array}$ & $\begin{array}{l}\mathrm{Cr} \\
\mathrm{Cl}\end{array}$ & $\begin{array}{l}\mathrm{Ar} \\
\mathrm{Bl}\end{array}$ \\
\hline
\end{tabular}

$\mathrm{A}=$ Guanethidine+Adrenaline, $\mathrm{B}=$ Guanethidine followed $30 \mathrm{~min}$ later by Adrenaline, $\mathrm{C}=$ Adrenaline followed $30 \mathrm{~min}$ later by Guanethidine, $r=$ right eye used as test, $l=$ left eye used as test

\section{Results and discussion}

Variation in IOP of rabbits' eyes

If the IOP of rabbits' eyes remained unchanged throughout a normal experimental period, or alternatively if the IOP changed in a systematic and reproducible manner, then the effect of drugs could be measured in both eyes, as a change in the expected IOP. To test this IOP measurements were made at intervals throughout the day on both eyes of all six rabbits. Measurements of this kind were carried out on four successive days, and the results for one rabbit are given in Fig. I.

Fig. I shows the IOP variation that was obtained in one rabbit during daylight hours on four successive days, and is typical of the results obtained with all six rabbits. It is quite clear that under our holding and experimental conditions no predictable or reproducible change in IOP occurs throughout the day.

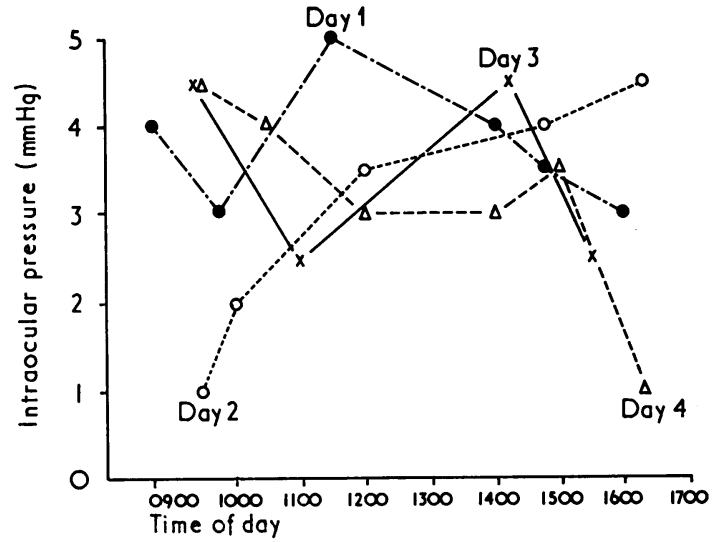

FIG. I Intraocular pressure variation in one rabbit during four successive days

\section{Normal variation in IOP between right and left eyes}

In order to test the efficiency of drugs in reducing IOP it was necessary to instill the drugs in one eye, using the other as the untreated control. For this to be justified it is necessary for the IOP of both untreated eyes of each rabbit to be similar. To test this, IOP measurements of all six rabbits were taken at intervals throughout each of four separate days. Fig. 2 shows the readings for the six rabbits on the same day.

As can be seen except in a few isolated cases the

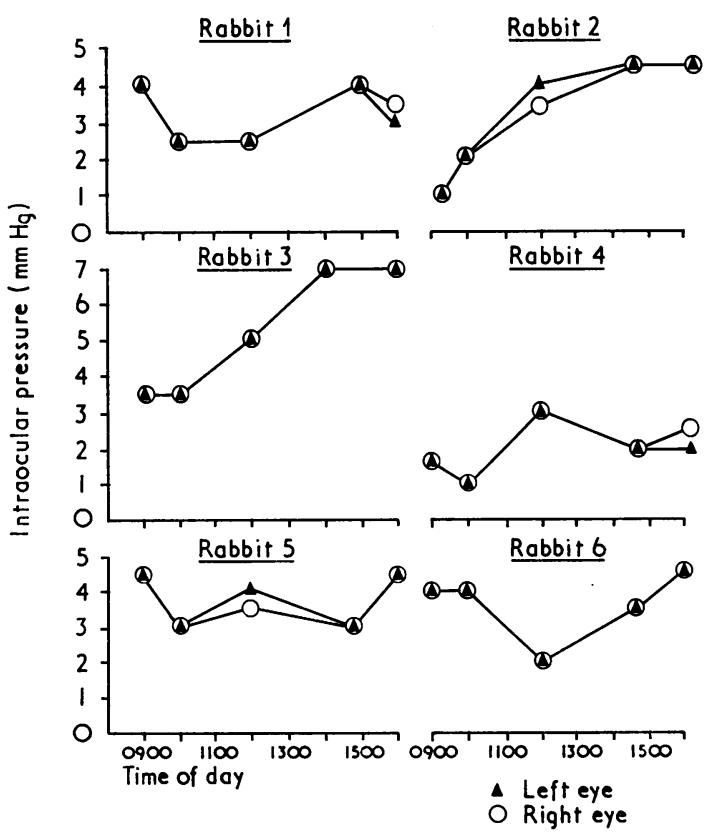

FIG. 2 Comparison of the intraocular pressure in the right and left eye of six rabbits during one day 
readings in the right and left eyes were the same. Therefore we feel justified in instilling the formulation into one eye, and using the difference in IOP between the test and control eye as a measure of the intensity of the effect. The duration of activity is given as the the time during which there is a measurable difference between the treated and control eyes. As all rabbits were used for all treatments the results obtained (in $\mathrm{mm}$ of $\mathrm{Hg}$ ) for both eyes in the six rabbits were totalled and expressed as the mean decrease in IOP.

\section{Effect of different dosage protocol on IOP}

Five different treatments were investigated, namely: Adrenaline 0.5 per cent; Guanethidine 2.5 per cent; Guanethidine followed 30 min later by Adrenaline; Adrenaline followed 30 min later by Guanethidine; Adrenaline and Guanethidine in the same formulation.

IOP readings were taken twice, at hourly intervals, before instillation and at 2, 5, 24, 29, 36, and 48 hours after instillation of the first drops. The results are shown in Fig. 3 and the mean IOP readings for the six rabbits together with their associated standard errors are given in Table II, for 2 and 5 hours after instillation of the drops. From Fig. 3 it appears that Guanethidine and Adrenaline given together produce a more intense effect which lasts longer than any of the other treatments.

Table II Mean IOP reduction with their associated standard errors for five experimental protocols in rabbits

\begin{tabular}{|c|c|c|}
\hline \multirow{2}{*}{$\begin{array}{l}\text { Dosage } \\
\text { protocol }\end{array}$} & \multicolumn{2}{|l|}{ Hours } \\
\hline & 2 & 5 \\
\hline $\begin{array}{l}\text { Adrenaline } \\
\text { Guanethidine }\end{array}$ & $\begin{array}{l}2 \cdot 5 \pm 1 \cdot 19 \\
2 \cdot 3 \pm 0 \cdot 90\end{array}$ & $\begin{array}{l}2 \cdot 7 \pm 0 \cdot 89 \\
2 \cdot 2 \pm 0 \cdot 67\end{array}$ \\
\hline $\begin{array}{l}\text { Adrenaline followed by } \\
\text { Guanethidine } \\
\text { Guanethidine followed by }\end{array}$ & $1 \cdot 6 \pm 0.64$ & $2 \cdot 4 \pm 0.62$ \\
\hline $\begin{array}{l}\text { Adrenaline } \\
\text { Adrenaline plus Guanethidine }\end{array}$ & $\begin{array}{l}2 \cdot 2 \pm 1 \cdot 09 \\
3 \cdot 2 \pm 1 \cdot 25\end{array}$ & $\begin{array}{l}2 \cdot 6 \pm 0 \cdot 36 \\
3 \cdot 8 \pm 1 \cdot 31\end{array}$ \\
\hline
\end{tabular}

The next most efficient treatment is provided by Guanethidine followed 30 min later by Adrenaline. Although the difference in initial intensity compared with the remaining three treatments is negligible, the duration of effectiveness is longer, the effect being well maintained at 36 hours. In contrast Adrenaline alone, while initially having a reasonable effect, has lost its action by 36 hours. All IOP measurements were back to normal after 48 hours.

Visually the results appear to be unequivocal with the peak of activity being obtained after about 5 hours. Checking experiments were performed on single rabbits when readings were taken after 7 and 9 hours which indicated that the IOP readings were beginning to rise again. The greatest difference at the 5 hour level occurs between Adrenaline plus Guanethidine and Guanethidine alone. A Student's ' $t$ ' test

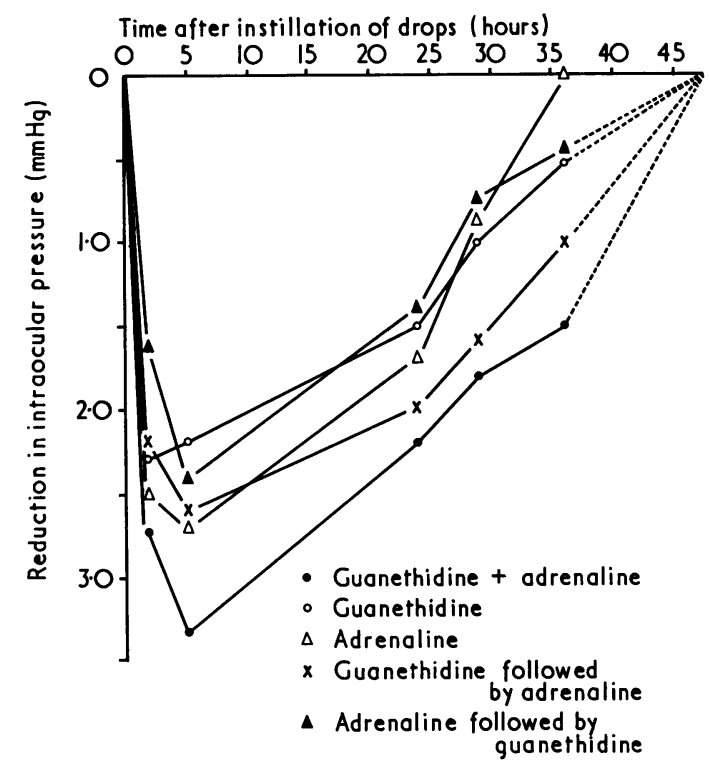

FIG. 3 Reduction in intraocular pressure brought about in rabbits by Guanethidine and Adrenaline given according to five different instillation protocols. Results are mean of those obtained with six rabbits

was carried out on these figures which gave a calculated value of 0.92 compared with the tabulated value of $I \cdot 73$ at the 5 per cent probability level. At the 2 hour level the same test was carried out for Adrenaline plus Guanethidine and Adrenaline alone and gave a value of $\mathrm{I} \cdot \mathrm{I} 4$, the tabulated value again being $\mathrm{I} \cdot 73$. Thus the readings obtained for Adrenaline plus Guanethidine cannot statistically be taken as being different from the others. This result is due to the high standard errors of all the values, which were about 30 to 50 per cent of their corresponding mean values in all cases. This is because we cannot measure the IOPs with a precision greater than $0.5 \mathrm{~mm}$ which is approximately 20 to 40 per cent of the experimental readings. In view of these constraints it is probably not justified to use normal statistical tests, and the most that can be concluded is that Guanethidine given with Adrenaline tends to give results at least as good as those obtained when the drugs are given separately.

Although Guanethidine followed by Adrenaline after $30 \mathrm{~min}$ is a standard treatment for glaucoma, the results with rabbits suggest that there is no advantage in administering the two types of drops separately. In view of the benefit to the patient obtained by giving both drugs in the same formulation it would appear sensible to attempt to treat patients with this combination.

Work undertaken in our laboratory has shown that there are no serious formulation problems associated with preparing a solution containing both drugs. The 
stability of the dual dosage is governed by the stability of the Adrenaline, and it is possible to prepare eye drops buffered to a $\mathrm{pH}$ of $7 \cdot 0$ to $7 \cdot 2$ with a shelf life at $4^{\circ} \mathrm{C}$ of greater than one year, so that it will subsequently remain stable during normal domiciliary use.

The formulation can be adequately preserved by the addition of 0.0 I per cent $w / v$ benzalkonium chloride and is best sterilized by filtration through a $0.22 \mu$ membrane.

The work, so far performed with rabbits, is encouraging. It is now necessary to subject eye drops containing I per cent Adrenaline and 5 per cent Guanethidine to extended trials on patients with chronic open-angle glaucoma.

\section{Summary}

The effect of Adrenaline 0.5 per cent and Guanethi- dine 2.5 per cent alone and in combination in reducing intraocular pressure in rabbits has been studied. Six rabbits were used and the efficiencies of 5 different dosage protocols was estimated both as intensity and duration of activity. The 5 dosage protocols were Adrenaline alone; Guanethidine alone; Adrenaline and Guanethidine together; Adrenaline followed in 30 min by Guanethidine; and Guanethidine followed in 30 min by Adrenaline. The single formulation containing both drugs appeared to be at least as efficient as the others. Eye drops containing I per cent Adrenaline and 5 per cent Guanethidine having sufficient stability for normal use have therefore been formulated.

This work was performed under the auspices of the Medical Research Council (Grant no. 7I5) to whom we should like to express our gratitude.

\section{References}

DUKE-ELDER, s. (1962) 'Sympathomimetic drugs' in 'System of Ophthalmology', vol. 7, p. 570. Kimpton, London

langham, M. E., kitazawa, y., and hart, R. w. (I97I) F. Pharmacol. exp. Ther., 179, 47

PATERson, G. D., and PATERSON, G. (1972) Brit. F. Opthhal., 56, 288

ROTH, J. A. (1973) Ibid., 57, 507

TURNer, P. (I968) In 'Evaluation of Drug Effects in the Eye', ed. P. V. Pigott, p. 7I. Trust for Education and Research in Therapeutics, London 